\title{
Dynamics of Induction and Expression of Long-Term Synaptic Facilitation in Aplysia
}

\author{
Juliane Mauelshagen, Gretchen R. Parker, and Thomas J. Carew \\ Department of Psychology, Yale University, New Haven, Connecticut 06520
}

Serotonin (5HT)-induced short-term facilitation and long-term facilitation (STF and LTF) of the monosynaptic connection between tail sensory neurons (SNs) and motor neurons (MNs) in Aplysia have been useful in delineating possible cellular mechanisms contributing to short-term and long-term memory. Previous work from our laboratory showed that LTF can be produced in the absence of STF, suggesting that these processes may be functionally independent. In the present study, we explored this hypothesis by examining the temporal relationship between STF and LTF. We recorded intracellularly from pairs of monosynaptically connected $\mathrm{SNs}$ and $\mathrm{MNs}$ in isolated pleural-pedal ganglia. In the first experimental series, we followed the time course of LTF across a $24 \mathrm{hr}$ period after its induction by five applications of $10 \mu \mathrm{M} 5 \mathrm{HT}$. STF completely decayed to baseline several hours before the expression of LTF. This biphasic expression profile of STF and LTF further supports the hypothesis that LTF is not a simple elaboration of STF. In the second experimental series, we monitored the immediate expression of facilitation during and after different numbers of $5 \mathrm{HT}$ applications. We identified a rapidly decaying STF (lasting 15-30 min) after one to four pulses of $50 \mu \mathrm{M} 5 \mathrm{HT}$ and a unique, prolonged intermediate-term facilitation (ITF; lasting up to $90 \mathrm{~min}$ ) after five pulses of $50 \mu \mathrm{M} 5 \mathrm{HT}$. These results raise the possibility that STF, ITF, and LTF may reflect components of different memory phases in the intact animal.

Key words: Aplysia; short-term facilitation; long-term facilitation; time course; sensory neurons; serotonin; memory induction; memory expression; memory phases
It has long been appreciated that memory can exist in many different forms ranging from short-term memory, lasting seconds to minutes, to long-term memory, lasting days, weeks, and even a lifetime. Considerable behavioral research has been devoted to identifying different phases of acquisition, storage, and retrieval that might give rise to these diverse expressions of memory. A particularly useful strategy in this kind of analysis has been to characterize the temporal dynamics of different phases of memory. This general approach has been applied successfully to many types of memory in a wide range of animals from invertebrate preparations, such as the honeybee (Hammer and Menzel, 1995) and Drosophila (deZazzo and Tully, 1995), to higher animals (McGaugh, 1966), including primates (Goldman-Rakic, 1992) and humans (Atkinson and Shiffrin, 1968). Elucidating the temporal dynamics of different phases of memory has helped to focus subsequent experimental efforts on specific time points when further analysis might reveal mechanistic differences in memory processing. By taking this type of analysis to the next step by examining different temporal phases of memory at a mechanistic level, it has become possible to begin to specify some of the critical cellular and molecular steps involved in different forms of memory processing in diverse species (for review, see Byrne, 1987; Hawkins et al., 1987; Squire, 1987; Dudai, 1989). One preparation that has been particularly useful for this type of analysis is the marine mollusc Aplysia, which displays several forms of associative and nonassociative memory that endure on time scales lasting

Received May 28, 1996; revised July 12, 1996; accepted Aug. 21, 1996.

This work was supported by a Deutsche Forschungsgemeinschaft grant to J.M. and National Institutes of Health Grant R01-MH-14-1083 to T.J.C. We thank Stephen Fisher, Eric Kandel, Sharen McKay, Carolyn M. Sherff, and Laura L. Stark for helpful comments on an earlier draft of this manuscript.

Correspondence should be addressed to Dr. Juliane Mauelshagen, Department of Psychology, Yale University, 2 Hillhouse Avenue, New Haven, CT 06520.

Copyright (C) 1996 Society for Neuroscience 0270-6474/96/167099-10\$05.00/0 from minutes to weeks (Carew and Sahley, 1986; Byrne, 1987; Hawkins et al., 1987).

The best characterized form of learning in Aplysia is sensitization, an increase in reflex amplitude after the presentation of a strong or noxious stimulus such as tail shock. Behavioral sensitization can exist in both short-term and long-term forms (Carew et al., 1971; Pinsker et al., 1973). Behavioral training that produces long-term sensitization also induces long-term facilitation (LTF) at the monosynaptic connections between identified sensory neurons (SNs) and motor neurons (MNs) in Aplysia (Frost et al., 1985). Comparable LTF can be produced in the intact CNS and in isolated cocultures of SNs and MNs by repeated application of serotonin (5HT), a transmitter released by tail shock (Montarolo et al., 1986; Mercer et al., 1991; Clark and Kandel, 1993; Emptage and Carew, 1993). 5HT is further implicated in behavioral sensitization, because systemic depletion of 5HT blocks the induction of reflex sensitization in Aplysia (Glanzman et al., 1989). With the $\mathrm{SN}-\mathrm{MN}$ synapse as a model system, considerable progress has been made in elucidating important synaptic, biophysical, and molecular steps involved in both short-term and long-termmemory in Aplysia (for review, see Byrne and Kandel, 1996; Carew, 1996).

Recently we have developed an experimental system, the tail SN-MN connection in the intact CNS, which allows a novel way of examining different phases of STF and LTF. In this preparation the SN synaptic terminals onto the MNs are far away from the SN cell body, providing sufficient spatial resolution to dissect out components of facilitation originating at the site of the synapse from those originating elsewhere, such as at the cell body. Using this experimental system, Emptage and Carew (1993) recently have shown that 5 HT-induced LTF can be expressed when shortterm facilitation (STF) is completely absent, indicating that STF and LTF may be induced and expressed independently and in 
parallel. In the present paper we further strengthen this hypothesis by showing that STF and LTF can be dissociated completely by their kinetics. First, using five pulses of $10 \mu \mathrm{M}$ 5HT, STF was induced and expressed immediately, but decayed back to baseline several hours before LTF was expressed. To further examine facilitation during and immediately after the induction of LTF, we next monitored the temporal expression of facilitation induced by different numbers of $5 \mathrm{HT}$ pulses. One to four pulses of $50 \mu \mathrm{M}$ 5HT induced STF that decayed rapidly (within 15-30 min), whereas five pulses induced a form of facilitation, intermediateterm facilitation (ITF), with a uniquely prolonged decay (up to 90 min), suggesting the existence of another, intermediate, form of synaptic facilitation induced by multiple applications of 5HT. Taken collectively, our results suggest that repeated exposure to 5HT induces multiple, overlapping, and perhaps functionally independent forms of facilitation at central synapses of Aplysia.

Some of these results have been reported previously in abstract form (Mauelshagen et al., 1995; Mauelshagen and Carew, 1996).

\section{MATERIALS AND METHODS}

\section{Preparation}

Wild-caught adult Aplysia californica (supplied from Marinus, Long Beach, CA, or Marine Specimens Unlimited, Pacific Palisades, CA) were anesthetized by injection of isotonic $\mathrm{MgCl}_{2}(100 \mathrm{ml} / 100 \mathrm{gm}$ of body weight). Single pleural-pedal ganglia were dissected and bathed for $45-60 \mathrm{sec}$ in $0.4 \%$ glutaraldehyde diluted in artificial seawater (ASW) containing (in mM): $460 \mathrm{NaCl}, 55 \mathrm{MgCl}_{2}, 11 \mathrm{CaCl}_{2}, 10 \mathrm{KCl}$, and 10 Tris, $\mathrm{pH} 7.4$, to reduce contraction of connective tissue during application of 5 HT. The ganglia were pinned in a SYLGARD-coated recording dish and desheathed to expose the somata clusters of tail SNs in the pleural ganglion and MNs in the pedal ganglion (Walters et al., 1983). Ganglia were prepared in a 1:1 mixture of ASW and isotonic $\mathrm{MgCl}_{2}$ to block synaptic transmission during dissection.

\section{Intracellular recordings}

During the experiment, the preparation was perfused with ASW (or 5HT in ASW) at room temperature $\left(20-22^{\circ} \mathrm{C}\right)$. The perfusion rate was adjusted to $\sim 5 \mathrm{ml} / \mathrm{min}$. The preparation was illuminated from below with a dark-field condenser. Tail SNs and tail MNs were identified by their location in characteristic somata clusters in the pleural and pedal ganglia, respectively. Electrodes for intracellular recordings had resistances that ranged between 8 and $15 \mathrm{M} \Omega$ with a $3 \mathrm{M} \mathrm{KCl}$ solution. Intracellular signals were amplified by an Axon 2 intracellular amplifier (Axon Instruments, Foster City, CA) or Getting intracellular amplifiers (model 5A). Data were recorded and analyzed by the "Spike" acquisition and analysis program (Hilal Associates, NJ; sampling rate $16 \mathrm{kHz}$ ).

Throughout the experiments, the SN resting potential was not manipulated experimentally, but the MNs were prevented from spiking by hyperpolarizing the neuron to approximately $-70 \mathrm{mV}$. The input resistance and resting potential of each neuron were checked frequently to judge the healthiness of the preparation. If any $\mathrm{SN}$ or $\mathrm{MN}$ had a resting potential below $-30 \mathrm{mV}$ or an input resistance below 20 or $10 \mathrm{M} \Omega$, respectively, the experiment was not continued $(\sim 25 \%$ of all preparations).

Monosynaptic EPSPs in the MN were evoked by 20 or $30 \mathrm{msec}$ depolarizing current pulses in the SN. At the beginning of the experiment, the amount of stimulating current in the SN was adjusted to evoke a single action potential; the current required to elicit an action potential ranged from 0.5 to $2 \mathrm{nA}$.

\section{Experimental protocol}

The time course of expression of STF and LTF was studied within two different time windows after the induction. In the first series of experiments, we examined the time course of facilitation across a $24 \mathrm{hr}$ period after induction of LTF by using five applications of 5HT (cf. Emptage and Carew, 1993). In the second series, we refined this analysis by monitoring more closely the time course of facilitation during its induction and immediate expression within the first $2 \mathrm{hr}$ after 5HT application. In this series, we also systematically examined the facilitatory effects of different numbers of $5 \mathrm{HT}$ applications (from 1 to 5).
Series I. The protocol for Series I is outlined in Figure 1. For the baseline, a nondepressed baseline EPSP amplitude was established by activating the SN three times at an interstimulus interval (ISI) of $15 \mathrm{~min}$. If any depression was observed $(>15 \%)$, the preparation was discarded ( $\sim 5 \%$ of all preparations). For induction, LTF was then induced by five applications of $10 \mu \mathrm{M} 5 \mathrm{HT}$ (6 min each) at an intertrial interval (ITI) of $15 \mathrm{~min}$. We used a concentration of $10 \mu \mathrm{M} 5 \mathrm{HT}$, because previous experiments showed that this concentration is sufficient to induce both STF and LTF (Emptage and Carew, 1993). In addition, this relatively low concentration (Clark and Kandel, 1993; Emptage and Carew, 1993) would reduce the possibility of obscuring any dynamics of the temporal profile of facilitation by satiating the response. Adhering to the $15 \mathrm{~min}$ ISI, we tested for STF within $30 \mathrm{sec}$ after the first 5HT application (short-term test). Then the microelectrodes were removed. After completion of the fifth 5HT application and subsequent washout, the perfusion was turned off, and the cells surrounding the SN and MN were marked by placing small crystals of DiI (Molecular Probes, Eugene, OR) onto the somata with a blunt microelectrode. In addition, a map of the relative positions of the neurons was drawn to facilitate the identification of the designated SN-MN pair at later test points. After allowing the dye crystals to dissolve into the membrane for $10-15 \mathrm{~min}$, the recording dish was immersed in a $50 \mathrm{ml}$ Pyrex dish containing ASW and stored at $15^{\circ} \mathrm{C}$ until the long-term test. The same pairs of neurons were reimpaled at either $3 \mathrm{hr}(n=12), 10 \mathrm{hr}(n=8), 15 \mathrm{hr}(n=6)$, or $20 \mathrm{hr}(n=9)$ after the completion of induction. Two long-term tests (ISI $=15 \mathrm{~min}$ ) were used to monitor the expression of LTF at the different time points. A separate control for the $15 \mathrm{hr}$ group was run without $5 \mathrm{HT}$ application $(n=6)$.

Series II. The protocol for Series II is outlined in Figure 4. These experiments were designed to trace the dynamics of synaptic plasticity during and immediately after the induction of LTF. Specifically, we were interested in two features of the synaptic plasticity: its magnitude and the time course of decay of STF before the later onset of LTF. A nondepressed baseline EPSP amplitude was established, as in Series I. Then 50 $\mu \mathrm{M} 5 \mathrm{HT}$ was applied either $1 \times(n=18), 2 \times(n=11), 3 \times(n=12), 4 \times$ $(n=9)$, or 5 times $(n=15 ; 5 \mathrm{~min}$ each $)$ at an ITI of $15 \mathrm{~min}$. We used a concentration of $50 \mu \mathrm{M}$ 5HT (compared with $10 \mu \mathrm{M}$ in Series I) so that the results of these experiments would be generally applicable to future experiments examining LTF induction by application of 5HT to only the synaptic region of the SNs [LTF requires $50 \mu \mathrm{M} 5 \mathrm{HT}$ when $5 \mathrm{HT}$ is restricted to the synaptic region; Emptage and Carew (1993); see also Clark and Kandel (1993)]. During the induction phase, the EPSP amplitude was recorded within $30 \mathrm{sec}$ after the first 5HT application to test for STF (Test I). To ensure that comparable STF was being examined across all groups, we discarded preparations that exhibited a $<40 \%$ increase above baseline in Test I ( $\sim 15 \%$ of all preparations). To prevent any activity-dependent facilitatory processes from being induced during the 5HT exposure (Hawkins et al., 1983; Walters and Byrne, 1983), we retested the EPSP only after the last $5 \mathrm{HT}$ application (Test II). To follow the immediate expression profile in each group, we activated the SN in 15 min intervals thereafter (in ASW) as long as the preparation was healthy (resulting in a variable number of preparations during later tests; $n \geq 6$ in all cases).

\section{Data analysis and statistics}

EPSP amplitudes were measured as the peak voltage of the EPSP. In cases of two summating EPSPs, if the first EPSP was still rising when the second started, the transition point between the first and second EPSP was taken as a conservative estimate of EPSP amplitude. For all experiments, the mean of the first three EPSP amplitudes was used as a baseline measure. All EPSP amplitudes were expressed as percentage of baseline. In Series I, the mean of the two long-term tests was used as the long-term test score. For statistical analysis, the median and interquartile ranges were calculated for each group of EPSPs. Overall differences within each group were determined first by a Friedman test. A Wilcoxon test subsequently was applied for pairwise within-group comparisons. A Kruskal-Wallis test was used for overall between-group comparisons. All tests were two-tailed.

\section{RESULTS}

At Aplysia tail SN-MN synapses, one application of 5HT produces STF, whereas five applications of 5HT induce both STF and LTF. Because LTF can be produced in the absence of STF, Emptage and Carew (1993) suggested that these processes may be ex- 


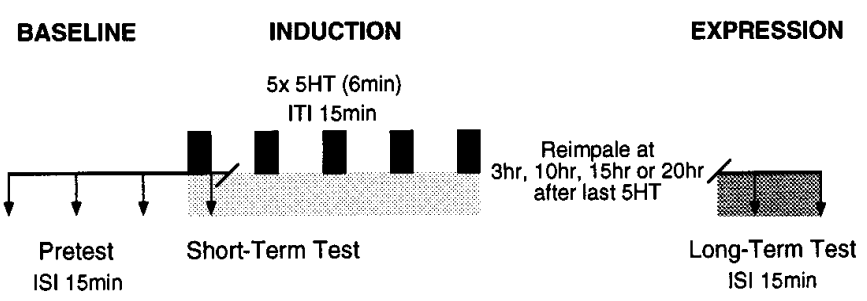

Figure 1. Experimental protocol for series I. A nondepressed baseline for the monosynaptic EPSP was established by activating the SN three times (Pretest) at an interstimulus interval (ISI) of $15 \mathrm{~min}$. Then LTF was induced by five $6 \mathrm{~min}$ applications of 5HT at an intertrial interval (ITI) of 15 min. STF was assessed immediately after the first 5HT pulse, and recording was stopped until the long-term test (broken line). The same SN-MN pair was reimpaled at the time points indicated to assess LTF expression by two long-term tests at an ISI of $15 \mathrm{~min}$.

pressed in parallel and independently. To further examine the relationship between these two forms of synaptic plasticity, we studied the time course of their expression in two different sets of experiments. In the first series, we determined the overall expression profile of LTF during a $24 \mathrm{hr}$ period after its induction by five applications of 5HT. In the second series, we examined the effect of different numbers of 5HT applications on the immediate expression profile of facilitation during the first $2 \mathrm{hr}$ after the $5 \mathrm{HT}$ administration.

\section{Series I}

\section{STF completely decays before the expression of LTF}

In this experimental series, LTF was induced by five applications of $10 \mu \mathrm{M}$ 5HT (Fig. 1). Each preparation was tested for STF after the first 5HT application and then was retested at either 3, 10, 15, or $20 \mathrm{hr}$ after the completion of induction. Figure $2 A-D$ shows examples of results for each experimental group. For example, in Figure $2 A$, clear STF was observed immediately after the first of five 5HT applications (light shading), but $3 \mathrm{hr}$ later, no synaptic facilitation was evident. This observation was striking, given the fact that this exact protocol of five exposures of 5HT is known to produce LTF $24 \mathrm{hr}$ later (Emptage and Carew, 1993). Thus, these results suggest that the short-term form of facilitation can decay completely before the expression of LTF. A similar result was obtained at the $10 \mathrm{hr}$ test. Clear STF was evident, but no facilitation was observed $10 \mathrm{hr}$ after $5 \mathrm{HT}$ (Fig. $2 \mathrm{~B}$ ). In contrast, in the 15 and $20 \mathrm{hr}$ tests, both STF and LTF (dark shading) were observed. In a control experiment (Fig. $2 E$ ) in which the 5HT applications were omitted, no changes in the EPSP amplitude with respect to baseline were observed when tested at time points corresponding to the short-term and $15 \mathrm{hr}$ tests.

A summary of all experiments is shown in Figure 3, in which the results of the short-term and long-term tests are expressed as median percentages of EPSP amplitudes and interquartile ranges with respect to baseline (100\%). For all four experimental groups, the Friedman test revealed overall significant differences among baseline, short-term, and long-term tests. Individual differences were analyzed further by the Wilcoxon test. During the induction phase, all groups showed significant STF with respect to baseline after the first pulse of 5HT ( $3 \mathrm{hr}: n=12, Z=2.93, p<0.003 ; 10$ hr: $n=8, Z=2.38, p<0.02 ; 15 \mathrm{hr}: n=6, Z=2.20, p<0.03$; $20 \mathrm{hr}: n=9, Z=2.67, p<0.008$; Wilcoxon test). There was no significant difference in STF among the groups $(\mathrm{df}=3, H=1.68$, $p=0.64$; Kruskal-Wallis test). However, for the long-term tests, there were pronounced differences among the relative EPSP amplitudes of the four groups $(\mathrm{df}=3, H=9.05, p<0.03)$. At 3 hr after the induction, the EPSP amplitude was recovered significantly, as compared with the short-term test $(n=12, Z=2.76$, $p<0.006)$ with no significant facilitation above baseline $(Z=$ $0.71, p=0.48$ ). Thus STF (as measured after the first 5HT application) has decayed completely by $3 \mathrm{hr}$ after 5HT administration, and any facilitation observed at later time points reasonably can be attributed to the expression of LTF (see Discussion). At $10 \mathrm{hr}$ after induction, there was again no significant facilitation above baseline $(n=8, Z=0.42, p=0.674)$. However, there was no significant difference between the short-term test and the $10 \mathrm{hr}$ test $(Z=0.98, p=0.33)$. As indicated by the large upper interquartile range, there was considerable variability in this group attributable to a subset of three of eight preparations in which the EPSP amplitude was elevated significantly above baseline levels at the $10 \mathrm{hr}$ test $(174,207$, and 343\%). This suggests that $\sim 10 \mathrm{hr}$ after induction there is a transition period during which LTF is just beginning to be expressed. Supporting this idea, significant facilitation above baseline levels was observed at $15 \mathrm{hr}$ after induction $(n=6, Z=2.20, p<0.03)$ that was comparable to levels of STF $(Z=0.73, p=0.46)$. Similarly, significant LTF was observed at $20 \mathrm{hr}$ after induction $(n=9, Z=2.07, p<0.04)$, which again was not significantly different from $\operatorname{STF}(Z=1.84$, $p=0.07)$. For the control group, no significant changes in the EPSP amplitude were observed at either the short-term or $15 \mathrm{hr}$ time points $\left(\mathrm{df}=2, \chi^{2}=2.33, p=0.31\right.$; Friedman test $)$. Thus, the increase in EPSP amplitude described in the above experiments is not attributable to a nonspecific increase in the EPSP occurring simply as a function of time. Rather, the increase is attributable to specific effects of 5HT on synaptic facilitation.

The data summarized in Figure 3 show that STF and LTF can be clearly dissociated in time: STF completely decays to baseline levels within $3 \mathrm{hr}$, whereas LTF is expressed significantly only at 15 and $20 \mathrm{hr}$. Previous results with this same protocol also reveal significant LTF at a $24 \mathrm{hr}$ test period (Emptage and Carew, 1993). Thus, in this system LTF is not a simple continuation or elaboration of STF but, rather, develops separately, with much slower kinetics beginning to be expressed $\sim 10 \mathrm{hr}$ after its induction.

\section{Series II}

From previous experiments it is known that a single application of $5 \mathrm{HT}$ induces STF, which declines to baseline levels within $\sim 15$ min, whereas five applications of 5HT induce LTF that lasts at least up to $24 \mathrm{hr}$ after its induction (Walters et al., 1983; Emptage and Carew, 1993; Stark et al., 1996). The data of Series I show that, with five applications of $10 \mu \mathrm{M}$ 5HT, STF decays to baseline levels within $3 \mathrm{hr}$ after $5 \mathrm{HT}$ application. In the next series of experiments, we wanted to examine systematically the time course of synaptic facilitation during the induction phase. In particular, we were interested in whether a different number of 5HT applications would have a differential effect on STF. To examine this question, we analyzed two aspects of STF: its magnitude and its time course of decay. The experimental procedure is illustrated in Figure 4. There were five experimental groups: the first received a single pulse of 5HT (50 $\mu \mathrm{M}$; see Materials and Methods), the second received two pulses (spaced by $15 \mathrm{~min}$, as in Series I), the third received three pulses, and so on. STF was examined after the first and (for 2-5× 5HT) after the last 5HT application (Test I and Test II, respectively), and the time course of decay of STF was monitored in $15 \mathrm{~min}$ intervals for the following $2 \mathrm{hr}$ (or as long as the preparation was viable). 

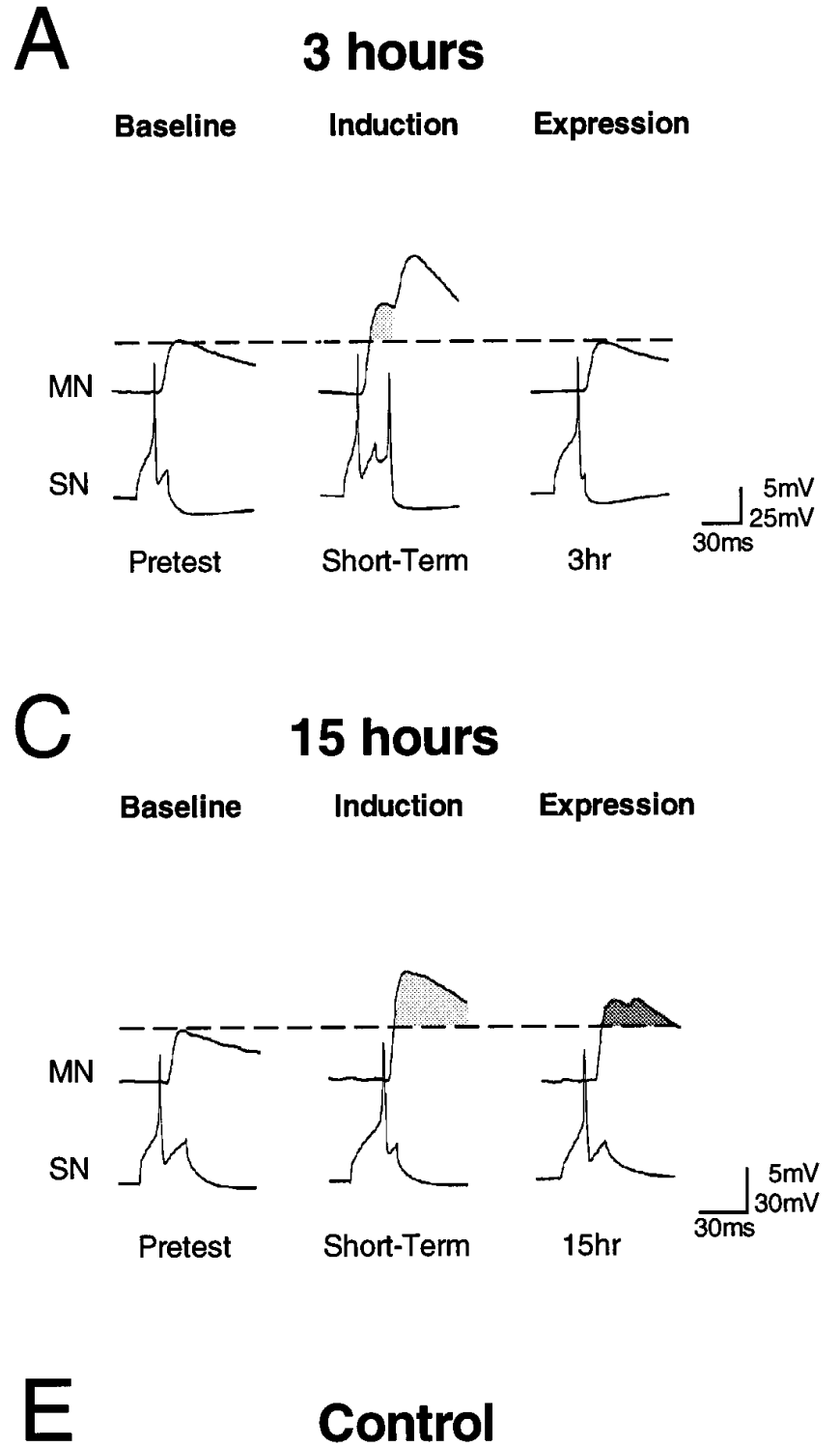

Baseline Induction Expression

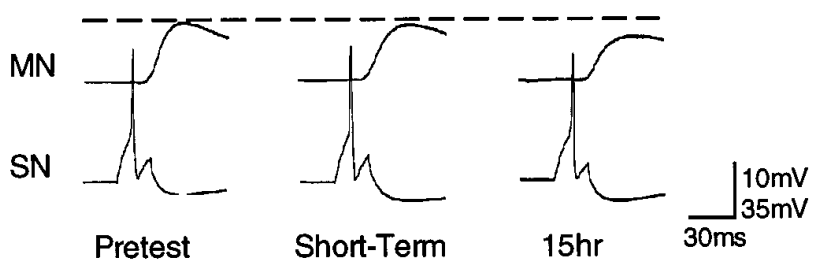

Different numbers of $5 H T$ pulses produce STF of comparable magnitude

The results of different numbers of $5 \mathrm{HT}$ pulses are shown in Figures $5 A-D(1-4 \times 5 \mathrm{HT})$ and $6(5 \times 5 \mathrm{HT})$. In each case a representative experiment is shown, together with a summary of all experiments of that group. To illustrate the time course of STF, the recording traces depict representative examples of one base-
B

10 hours

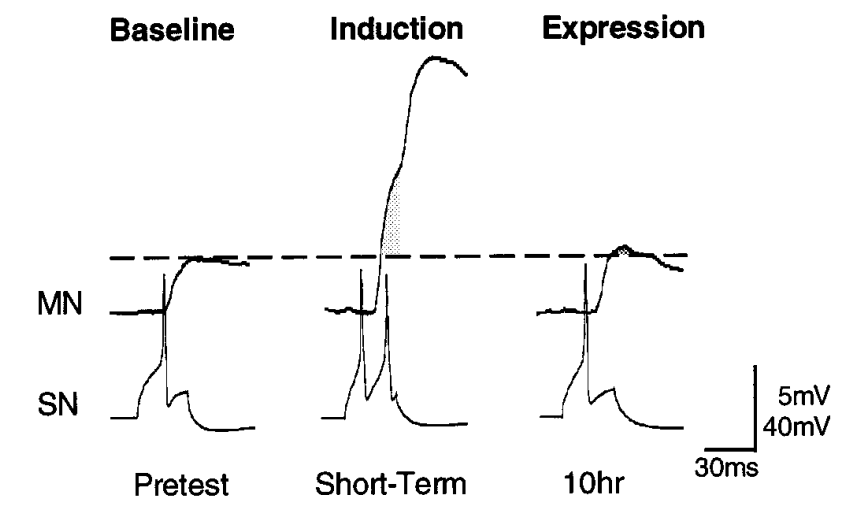

D

\section{0 hours}

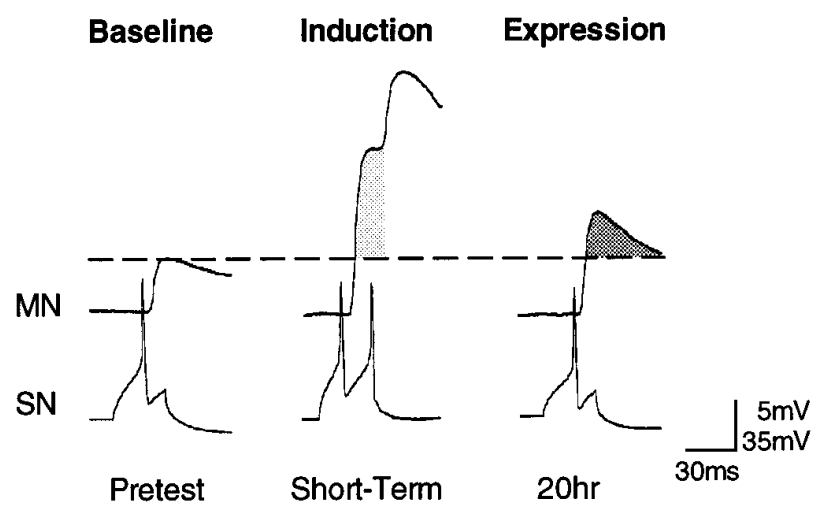

Figure 2. Representative recording traces for four experimental groups $(A-D)$ in which LTF was induced by five 5HT applications and a control group $(E)$, which did not receive any $5 \mathrm{HT}$. The monosynaptic EPSP in the $M N$ was evoked by a $20 \mathrm{msec}$ depolarization of the $S N$. For each group, the traces depict one of the three baseline stimulations (Pretest), the short-term test after the first of five 5HT applications (Short-Term), and one of the long-term tests at the time indicated. The stippled lines mark the baseline EPSP amplitude. STF and LTF above baseline are represented by light and dark shading, respectively. Note that STF was, in general, accompanied by increased excitability in the $S N$, but LTF was not.

line, Tests I and II, and the tests at 30, 60, and 120 min after the last 5HT application. In the summary graphs, the median percentage of EPSP amplitudes and interquartile ranges with respect to baseline are illustrated for each of the test points. Again for all groups, a Friedman test confirmed overall differences across the different tests (data not listed). Pairwise within-group comparisons showed that all groups exhibit significant STF at Test I and 


\section{3 hours $\quad 10$ hours $\quad 15$ hours 20 hours Control}

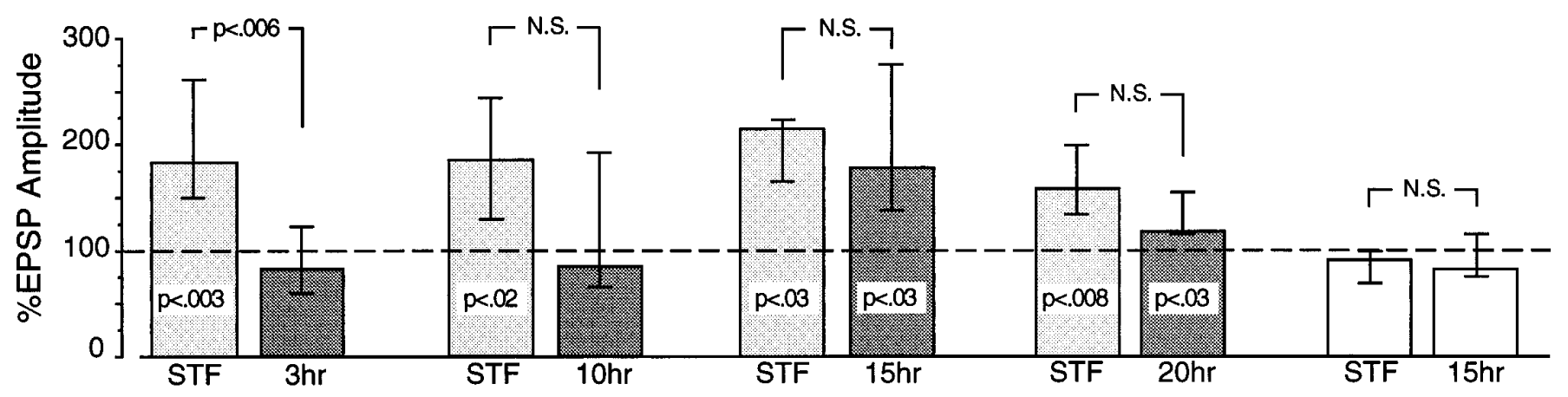

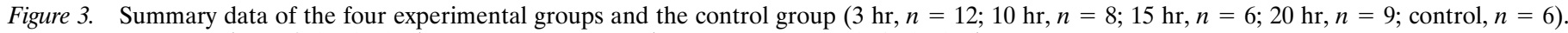

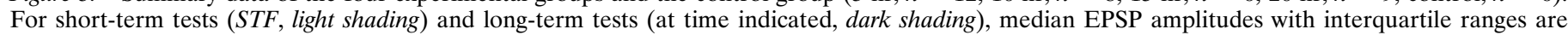
expressed relative to baseline (100\%, stippled line). $p$ within the bars indicates significant facilitation above baseline.

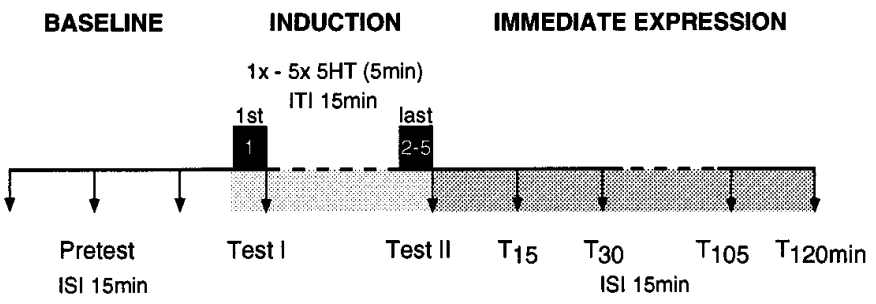

Figure 4. Experimental protocol for series II. A nondepressed baseline for the monosynaptic EPSP was established by three SN stimulations (Pretest) at an interstimulus interval (ISI) of $15 \mathrm{~min}$. In five experimental groups, one, two, three, four, or five $5 \mathrm{~min}$ applications of $5 \mathrm{HT}$ were administered at an intertrial interval (ITI) of $15 \mathrm{~min}$. In all groups, STF was assessed immediately after the first $5 \mathrm{HT}$ pulse (Test $I$ ) and, for groups with two to five $5 \mathrm{HT}$ applications, immediately after the last $5 \mathrm{HT}$ pulse (Test II). The immediate expression of facilitation was monitored in 15 min intervals thereafter.

Test II with respect to baseline $(1 \times 5 \mathrm{HT}-$ Test I: $n=18, Z=$ 3.72, $p<0.0002 .2 \times 5$ HT-Test I: $n=10, Z=2.80, p<0.005$; Test II: $n=11, Z=2.93, p<0.003 .3 \times 5$ HT-Test I: $n=11, Z=$ 2.93, $p<0.003$; Test II: $n=12, Z=2.59, p<0.01$. $4 \times 5$ HT-Test I: $n=9, Z=2.67, p<0.008$; Test II: $n=9, Z=2.55, p<0.01$. $5 \times 5$ HT-Test I: $n=15, Z=3.41, p<0.0007$; Test II: $n=15$, $Z=2.78, p<0.006$; Wilcoxon test). Moreover, after three, four, or five applications of 5HT, the amount of facilitation at Test II was found to be significantly smaller than during Test I $(3 \times 5 \mathrm{HT}$ : $Z=2.67, p<0.008 ; 4 \times 5 \mathrm{HT}: Z=2.55, p<0.01 ; 5 \times 5 \mathrm{HT}: Z=$ $2.22, p<0.03$ ). Thus, during the induction phase of LTF, repeated applications of 5HT do not result in increasing amounts of facilitation, i.e., there is no additive or summating effect of multiple 5HT pulses on the amount of STF. Rather, with three or more applications, facilitation becomes somewhat reduced after the last application, as compared with the first. A between-group comparison revealed that the amount of facilitation at Test I and Test II was comparable among the five groups; thus, there was no significant difference among them (Test I: $\mathrm{df}=4, H=3.91, p=$ 0.42; Test II: df $=4, H=3.73, p=0.45$; Kruskal-Wallis test).

One to four pulses of 5 HT produce STF with a rapid decay, whereas five pulses produce a uniquely prolonged decay

For all groups with $1-4 \times 5 \mathrm{HT}$, the time course of the immediate expression was very short-lasting (Fig. 5). After $1 \times$ and $2 \times 5 \mathrm{HT}$, the EPSP amplitude was facilitated significantly for only $15 \mathrm{~min}$ and decayed to baseline within $30 \mathrm{~min}$ after the last 5HT application $(1 \times 5 \mathrm{HT}-\mathrm{T} 15$ min: $n=18, Z=2.373, p<0.018$; T30 $\min : n=13, Z=0.25, p=0.81 .2 \times 5 \mathrm{HT}-\mathrm{T} 15 \mathrm{~min}: n=10, Z=$ 2.293, $p<0.022$; T30 min: $n=9, Z=0.42, p=0.67)$. After $3 \times$ and $4 \times 5 \mathrm{HT}$, STF decayed even more rapidly; there was no significant facilitation observed beyond Test II $(3 \times 5 \mathrm{HT}-\mathrm{T} 15$ $\min : n=12, Z=1.49, p=0.14 .4 \times 5 \mathrm{HT}-\mathrm{T} 15 \min : n=8, Z=$ $1.54, p=0.12$ ). In contrast, five applications of 5HT induced a much longer-lasting immediate expression (Fig. 6), with significant facilitation above baseline levels observed up to $90 \mathrm{~min}$ after the last 5HT application (T15 min: $n=14, Z=2.79, p<0.005$; T30 min: $n=11, Z=2.67, p<0.008$; T45 min: $n=9, Z=2.52$, $p<0.01$; T60 min: $n=7, Z=2.37, p<0.02$; T75 min: $n=7, Z=$ 1.86, $p=0.06$; T90 min: $n=6, Z=1.99, p<0.05)$. This prolonged phase of facilitation does not develop gradually with an increasing number of 5HT applications but seems to be switched on only by the fifth pulse of $5 \mathrm{HT}$.

Because 5HT is bath-applied in these experiments and because STF is likely induced at the synaptic terminals of the SNs (see Discussion), the question could be raised as to whether the emergence of the enhanced time course of facilitation after five pulses of 5HT (Fig. 6) simply might reflect the eventual penetration of 5HT to the terminal region (in sufficient concentration to increase the STF process). This interpretation is unlikely because, if the 5HT concentration at the synapse would increase progressively with repeated exposure to 5HT, one would expect a graded enhancement of the amplitude (Stark et al., 1996) and duration of STF after each additional 5HT pulse. However, this is not observed. Rather, we observe a slight decrease in the amplitude of facilitation after $3-5 \times 5 \mathrm{HT}$ (Test II) and a decrease in duration of STF after $3 \times$ and $4 \times 5 \mathrm{HT}$. Only after $5 \times 5 \mathrm{HT}$ is the duration of facilitation prolonged abruptly and significantly. Thus, the data are inconsistent with the interpretation that gradual and cumulative 5HT penetration with increased pulses of 5HT contributes significantly to the results we observe.

In considering the pronounced enhancement of STF duration produced by $5 \times 5 \mathrm{HT}$, it is not obvious from the data whether the rapid decay found with $1-4 \times 5 \mathrm{HT}$ is being prolonged precipitously by the fifth pulse of $5 \mathrm{HT}$ or whether an additional facilitatory process is initiated independently by the fifth pulse that is superimposed on the short-term decay. Additional analysis of the 
A

$1 \times 5 H T$
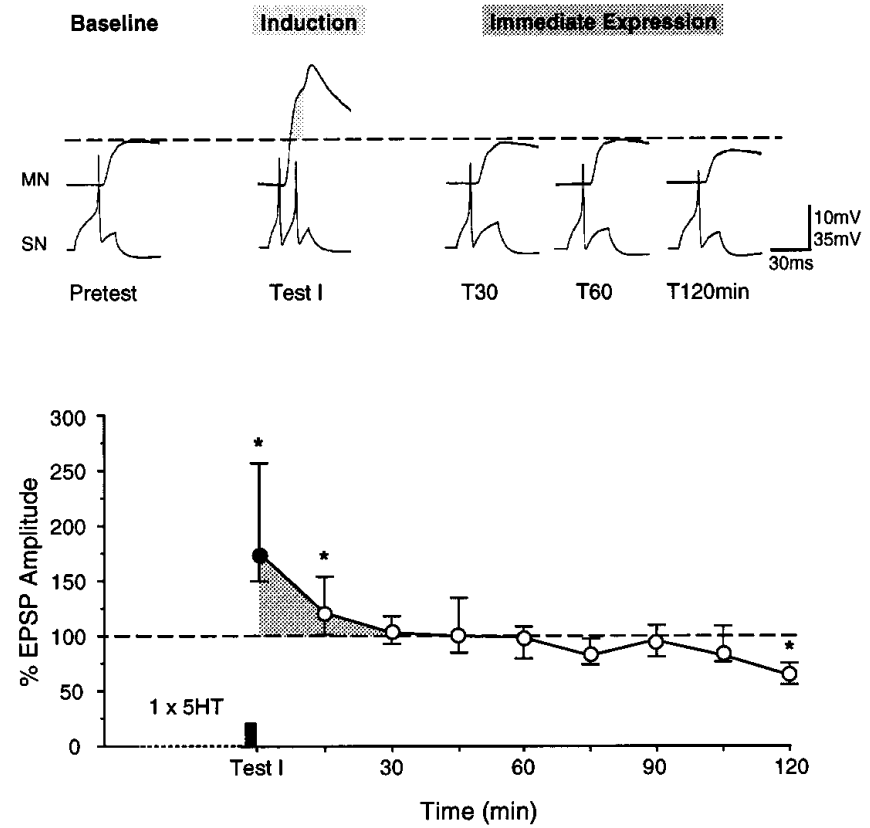

C

$3 \times 5 \mathrm{HT}$
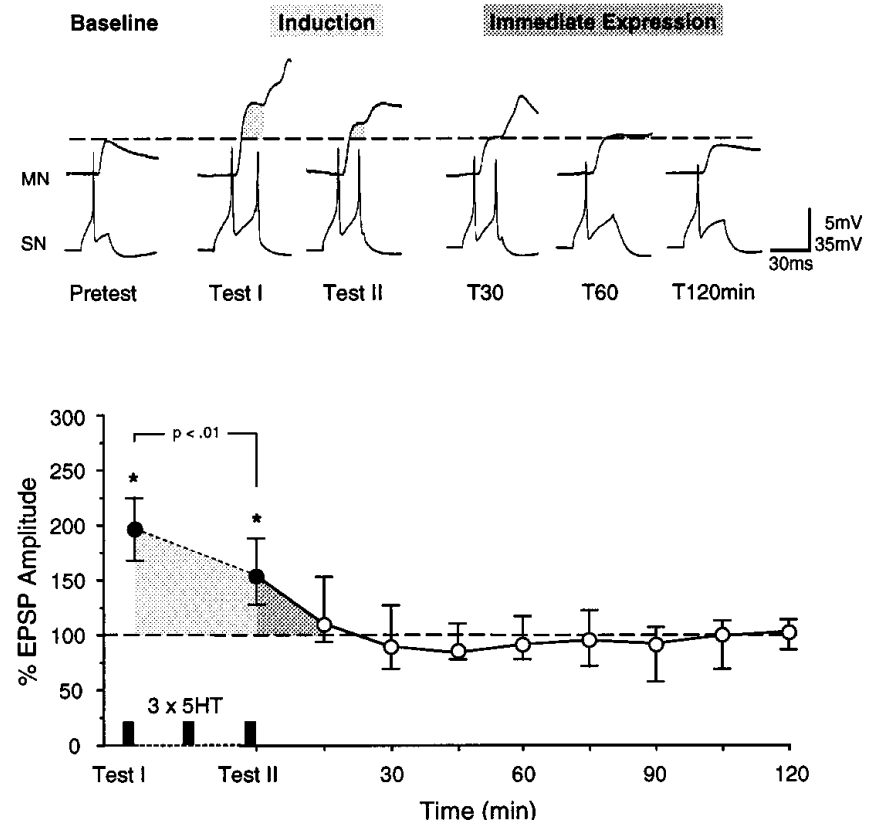

\section{$2 \times 5 H T$}
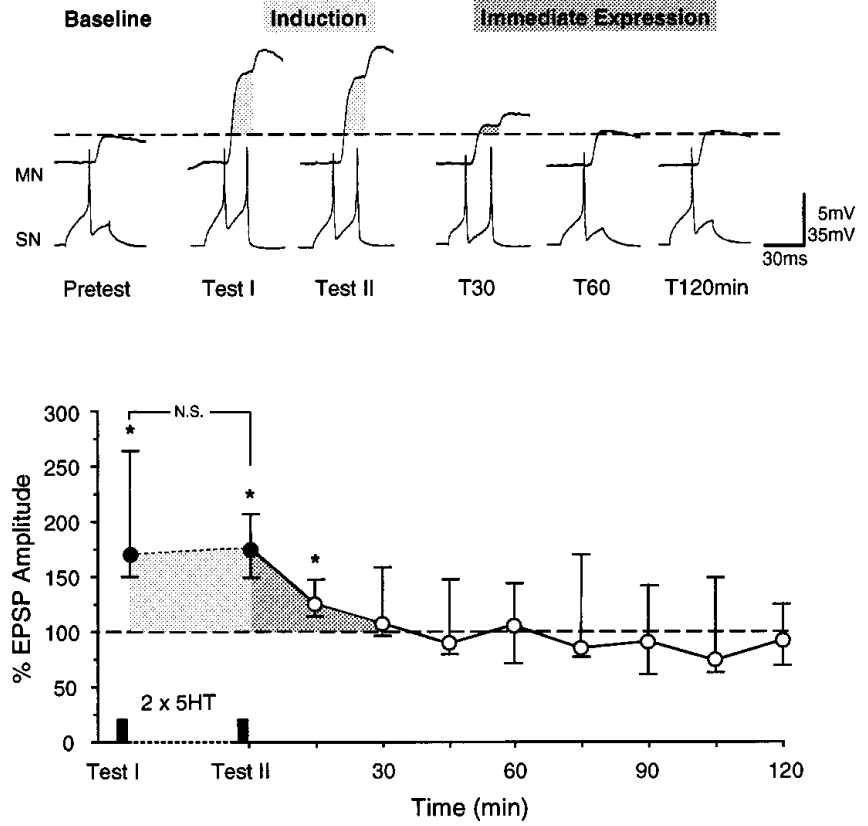

$4 \times 5 H T$
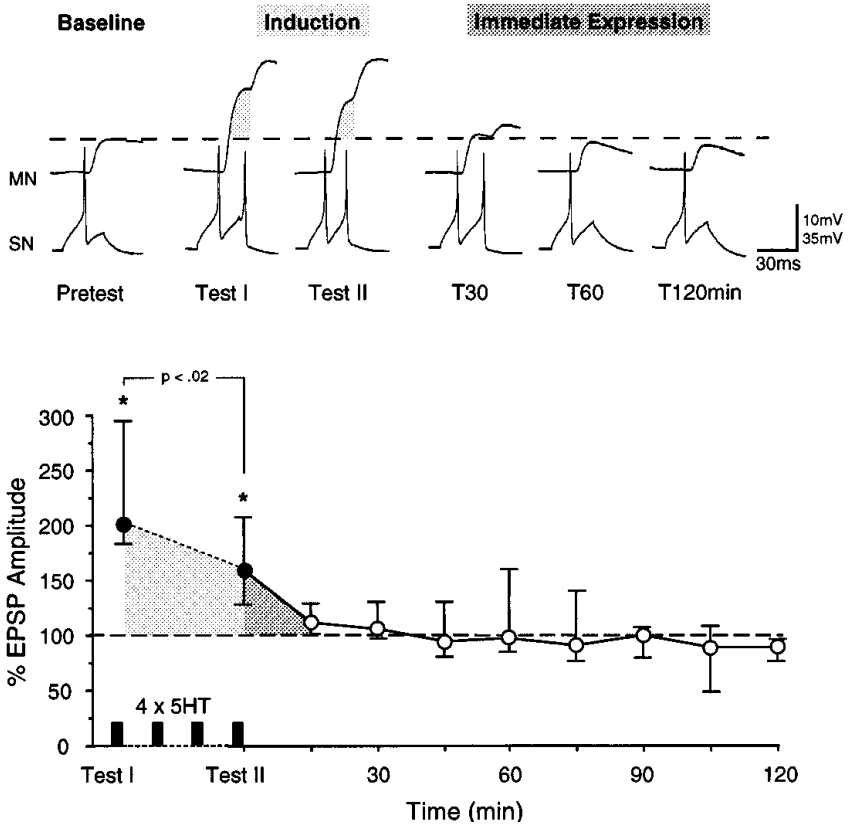

Figure 5. Recording examples and summary graphs for the experimental groups receiving $1 \times(n=18), 2 \times(n=11), 3 \times(n=12)$, or $4 \times 5 \mathrm{HT}(n=9$; $A-D)$. The monosynaptic EPSP in the MN $(M N)$ was evoked by a $30 \mathrm{msec}$ depolarization of the SN (SN). For each group, the traces depict one of the three baseline stimulations (Pretest), the short-term tests after the first (Test I) and, for groups $2-5 \times$, after the last 5HT application (Test II), and, finally, the tests at 30,60, and 120 min after the last 5HT pulse. The stippled lines mark the baseline EPSP amplitude. STF and LTF above baseline are represented by light and dark shading, respectively. Note that STF was accompanied by increased excitability in the SN that, in the cases of multiple 5HT applications, lasted for at least $30 \mathrm{~min}$. The graphs summarize results for each test point (Test I and Test II and tests across a 2 hr period thereafter). Median EPSP amplitudes with interquartile ranges are expressed relative to baseline (100\%, stippled line). Light and dark shading indicates significant facilitation above baseline during the induction and immediate expression phase, respectively. Because recording over the entire time period was technically not always possible, the $n$ varies at later test points ( $n \geq 6$ in all cases). ${ }^{*} p<0.05$; see Results. 

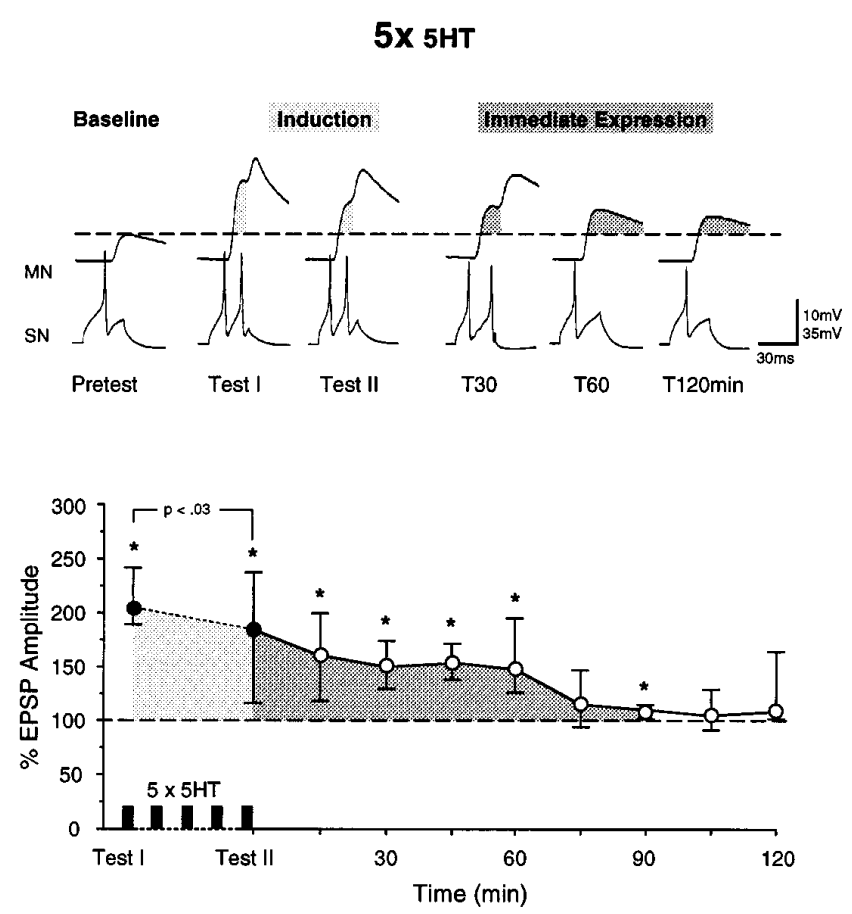

Figure 6. Recording examples and summary graph for the experimental group receiving $5 \times 5 \mathrm{HT}(n=15)$. Data are expressed as described in Figure 5.

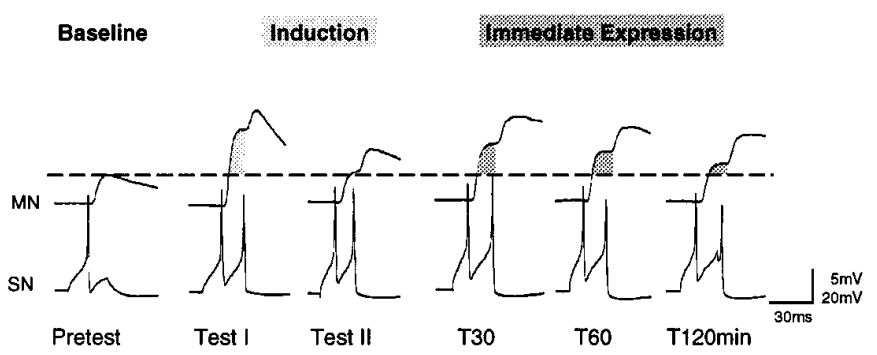

Figure 7. Example of a subset of preparations that showed a clear biphasicity after receiving five pulses of 5HT (compare with Fig. 6). Data are expressed as in Figures 5 and 6.

data favors the latter possibility. For $5 \times 5 \mathrm{HT}$, at Test II, the variance of observed EPSP amplitudes is very large, as compared with Test I or later tests (e.g., T45 min, T60 min). This reflects the fact that, at Test II in the $5 \times 5$ HT group, in some preparations the EPSP amplitude had decayed back to near baseline, whereas in some other preparations the EPSP amplitude even exceeded the value of Test I. However, in all preparations, the EPSP was facilitated at subsequent test points. These observations suggest that there may be more than one process responsible for shaping the EPSP amplitude across time after five pulses of 5HT. In fact, in a few recordings, we found a pronounced biphasicity in the decay kinetics, suggesting that the prolonged STF induced by five pulses (Fig. 6) may be a parallel process that is switched on during or some time after the last 5HT application. An example of such a biphasic facilitation profile is shown in Figure 7, in which, after five pulses of 5HT, the EPSP amplitude returned to baseline levels at Test II but subsequently increased again, expressing an apparently separate phase of facilitation across later test points. Consistent with this view, in all cases in which this biphasic profile

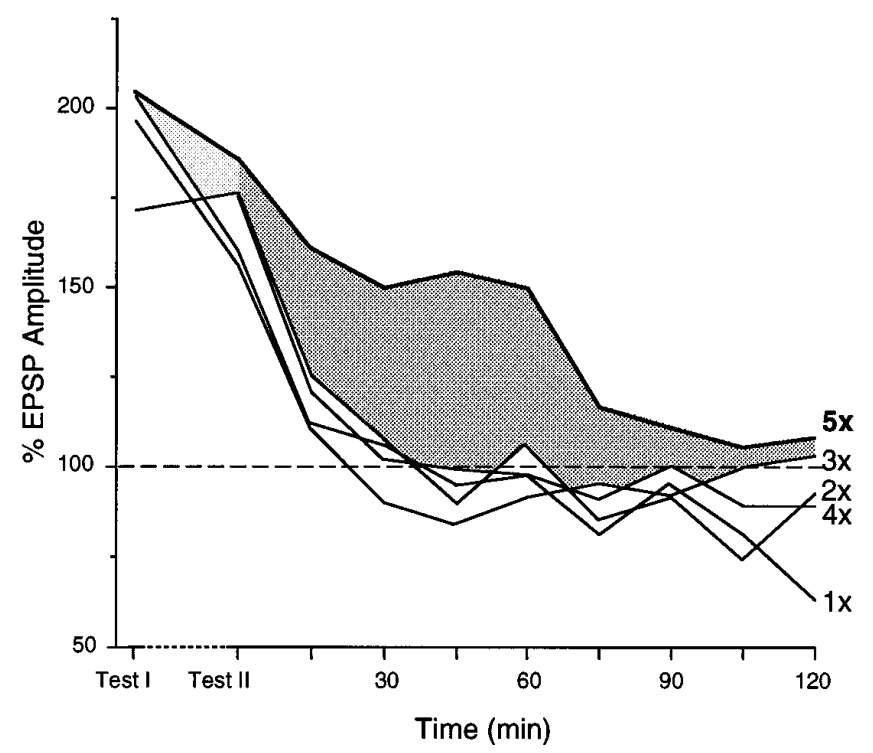

Figure 8. Comparison of time course of decay among the five experimental groups. The medians (without interquartile ranges) of the relative EPSP amplitudes are superimposed for all test points. The stippled line marks the baseline EPSP amplitude. The shading emphasizes the difference in decay kinetics between $5 \times 5 \mathrm{HT}$ and $1-4 \times 5 \mathrm{HT} .5 \times 5 \mathrm{HT}$ produces a significantly prolonged decay, as compared with all other groups, which do not differ among themselves (see Results).

was observed, the second phase of facilitation had a prolonged time course comparable to that shown in Figure 6.

A summary of all results in Series II is shown in Figure 8, which superimposes the time course of STF of all groups. The data shown in this manner emphasize the similarity between the rapid decay of STF for groups receiving one to four pulses of 5HT and the unique prolonged decay of facilitation induced by five pulses of 5HT. An overall between-group comparison reveals that five pulses of 5HT produce a significantly extended decay of facilitation, as compared with all other groups $(\mathrm{df}=4 ; F=3.07 ; p<$ 0.04 ; ANOVA), which do not differ from one another $(\mathrm{df}=3 ; F=$ $0.66 ; p=0.59)$. Thus, the results suggest that the fifth pulse of $5 \mathrm{HT}$ produces a unique form of synaptic facilitation characterized by a significantly prolonged time course of decay.

\section{DISCUSSION}

\section{Functional dissociation of STF and LTF}

In this paper we were interested in applying a common strategy in memory research: examination of the temporal dynamics of different phases of memory (McGaugh, 1966; Atkinson and Shiffrin, 1968; Goldman-Rakic, 1992; deZazzo and Tully, 1995; Hammer and Menzel, 1995) to a specific set of synapses in Aplysia that are known to be altered in the expression of both short-term and long-term memory (Walters et al., 1983; Frost et al., 1985). We show that, in Aplysia tail sensory-motor synapses, two forms of synaptic plasticity, STF and LTF, can be clearly dissociated by their kinetics: STF completely decays several hours before the expression of LTF begins. This biphasic expression profile of STF and LTF suggests that LTF is not a simple elaboration of STF, adding further support to the idea that STF and LTF at these synapses may be functionally independent. This hypothesis is based primarily on the finding by Emptage and Carew (1993) that LTF induction at tail sensory-motor synapses does not require the expression of 
STF. In their experiments, STF expression was prevented in three different ways: (1) by blocking STF with the 5HT antagonist cyproheptadine (Mercer et al., 1991), (2) by using 5HT concentrations subthreshold for the expression of STF, and (3) by applying $5 \mathrm{HT}$ exclusively to the SN somata, but not their terminals, onto tail MNs. In all groups, LTF was, nonetheless, expressed $24 \mathrm{hr}$ later. These results imply that STF requires $5 \mathrm{HT}$ to act directly on the SN terminals where covalent modifications modulate transmitter release (for review, see Byrne and Kandel, 1996). In contrast, the signaling cascade for LTF induction involves new protein synthesis (Montarolo et al., 1986; Ghirardi et al., 1995) and can be accessed directly within the SN somata or alternatively by a retrograde message from the synapse (Clark and Kandel, 1993; Emptage and Carew, 1993). The delayed onset of LTF (of 10-15 hr) that we observe likely reflects the total time required for 5HT-induced protein synthesis, subsequent transport of proteins to the SN terminal region, and the induction of structural changes at the synapse (Bailey and Chen, 1988). In cultured SNs, the critical time window for protein synthesis lasts only up to $2 \mathrm{hr}$ after $5 \mathrm{HT}$ application (Montarolo et al., 1986). If the same rules apply in the intact ganglion, the delayed onset of LTF in our experiments should be attributable primarily to the time needed for the transport of newly synthesized proteins $(\sim 50 \mathrm{~mm} / \mathrm{d}$ fast anterograde transport; Ambron et al., 1974) and the structural remodeling of the synapse (Bailey and Chen, 1988).

Ghirardi et al. (1995) recently provided a systematic study of both time and concentration dependence of STF and LTF in cultured SNs and MNs of Aplysia. In contrast to our data, these authors do not report a biphasic expression profile for STF and LTF. Rather, they observed maximal facilitation at 30 min after the last of five 5HT applications, with an initially fast decay followed by an asymptotic expression profile during the ensuing $24 \mathrm{hr}$ period. One likely explanation for the differences in the time course between the results of Ghirardi et al. (1995) and our results is that, in cell cultures of Aplysia neurons, the distance between the SNs and MNs is much shorter than in the intact ganglion. If, as discussed above, the rate-limiting step in LTF expression is the transport of newly synthesized proteins from the nucleus to the terminals, one would expect a much faster expression of LTF in cultured neurons. Likewise, a retrograde messenger from the synapse should be able to pass the distance to the SN soma with relatively little delay. Thus, in the experiments of Ghirardi and colleagues (1995), because of the short distance from soma to terminal, STF and LTF processes are likely to overlap to a high degree, whereas in the intact ganglia this distance is much greater, giving rise to temporal separation of STF and LTF.

\section{Decay kinetics of STF}

A surprising finding of our experiments was the uniquely prolonged duration of facilitation observed after five applications of 5HT: STF as produced by 1-4 5HT applications decayed to baseline within 15-30 min, whereas five 5HT applications induced a form of facilitation that lasted up to $90 \mathrm{~min}$. This prolonged form of facilitation did not develop gradually with an increasing number of 5HT applications but seemed to be switched on only by the fifth 5HT pulse. This observation raises the possibility that there may be two different processes involved in shaping the immediate expression of facilitation: one (STF) that undergoes a monotonic decrease with repeated 5HT applications and decays within 15-30 min and another (ITF) that requires repeated expo-

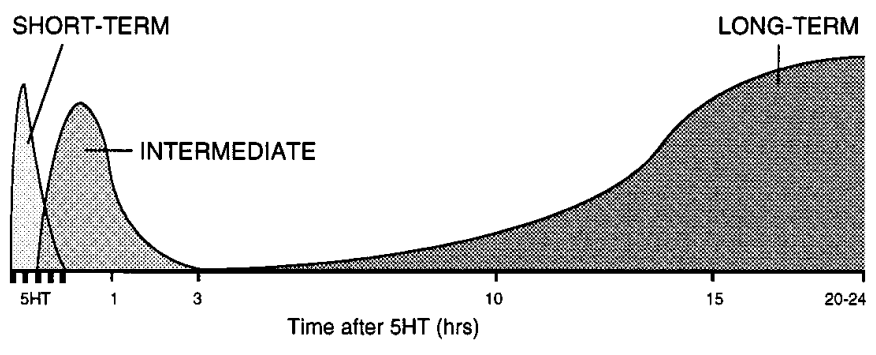

Figure 9. Hypothetical time course of three different phases of facilitation induced by multiple applications of 5HT. Short-term facilitation (STF) is maximally expressed immediately after the first $5 \mathrm{HT}$ pulse and declines with repeated exposure to 5HT. Overlapping with the decay of STF, an intermediate-term facilitation emerges that decays within 1-3 hr after the last $5 \mathrm{HT}$ application. Long-term facilitation is not expressed before $10 \mathrm{hr}$ after the 5HT exposure and lasts for at least $24 \mathrm{hr}$.

sure to 5HT to be initiated and does not decay until the following 1-2 hr. In Figure 9 the inferred time courses of STF, ITF, and LTF are summarized in schematic form.

At least two important questions arise from the model proposed in Figure 9. First, is ITF necessary for or supportive of LTF induction? LTF induction requires protein synthesis up to $2 \mathrm{hr}$ after the last 5HT application (Montarolo et al., 1986). Presumably, it takes several hours to transport newly synthesized proteins to the terminals (which are typically at least several millimeters away) and even more time if LTF is induced via a retrograde messenger from the terminals $[\sim 50 \mathrm{~mm} / \mathrm{d}$ fast anterograde transport, Ambron et al. (1974); $\sim 25 \mathrm{~mm} / \mathrm{d}$ retrograde transport, Ambron et al. (1992)]. The immediate expression of ITF without a time delay indicates that it relies predominantly, if not exclusively, on processes within the SN terminals. Also, any kind of a retrograde messenger that could be produced in the terminals during ITF expression would arrive at the SN soma too late to affect directly the protein synthesis required for LTF induction. Although the lack of dependence of LTF on STF (Emptage and Carew, 1993) suggests that ITF expression is also not necessary for LTF induction, it is still possible to envision a supportive role of ITF for LTF. For example, ITF could induce some transient changes at the terminals that, at a time when new proteins arrive from the SN soma, could provide an easier or faster restructuring of the synaptic terminal region, thereby enhancing the probability or amount of ultimate LTF expression. Moreover, such a sitespecific production of LTF may provide one possible way of providing synapse-specific long-term plasticity, as opposed to cell-wide changes (Clark and Kandel, 1993; Emptage and Carew, 1993). Finally, our data cannot rule out the possibility that, although STF is not required for LTF at these synapses, ITF may play some essential role in inducing LTF.

A second interesting question concerns the mechanisms of ITF. An intermediate-term facilitation has been reported previously by Ghirardi et al. (1995). Examining the time and concentration dependence of STF and LTF in dissociated cell culture, they found that both processes were recruited as a function of $5 \mathrm{HT}$ concentration. The differential dependence on 5HT concentration allowed a dissection of the overall temporal expression profile into two phases, an ITF lasting between $<3-6 \mathrm{hr}$ and an LTF lasting $24 \mathrm{hr}$. Interestingly, at concentrations that were just subthreshold to produce LTF, ITF depended exclusively on translation, but not transcription, of new protein. One intriguing question arising from this result is whether ITF in our preparation also might depend on translation of new 
proteins. If this were the case, because of the distance from the SN soma, the immediate expression of ITF would require a pool of messenger RNA and translation machinery (e.g., ribosomes) close to the synaptic terminal region. Selective transport of mRNAs to specific synaptic sites has been reported for vertebrate neurons, but only for postsynaptic sites of plasticity (Steward and Banker, 1992). Considering these data, we should not exclude the postsynaptic site as a possible origin of some aspects of intermediate and long-term synaptic facilitation in Aplysia.

\section{Behavioral implications}

Our present findings taken with previous results (Montarolo et al., 1986; Emptage and Carew, 1993; Ghirardi et al., 1995; Mauelshagen et al., 1995, 1996) suggest that 5HT can induce different, functionally independent forms of synaptic plasticity that may be dissociated by their time course, by their dependence on concentration, duration, and pattern of 5HT application, and by their intracellular mechanisms. On the basis of the assumption that 5HT-induced facilitation plays a major role in behavioral sensitization (Brunelli et al., 1976; Walters et al., 1983; Abrams et al., 1984; Glanzman et al., 1989), it is possible that the behavioral retention profile for sensitization is also composed of multiple phases, depending on the pattern and intensity of training stimuli. Current work in our laboratory is aimed at examining whether short-term and long-term memory for behavioral sensitization in Aplysia may, in fact, be composed of multiple, dissociable phases, as suggested in Figure 9. Multiphasic memory processing has been suggested for many other vertebrate and invertebrate systems (Kamin, 1957; McGaugh, 1966; Gibbs and Ng, 1979; Rosenzweig and Bennett, 1984; deZazzo and Tully, 1995; Hammer and Menzel, 1995). Two recent studies are especially relevant to this issue, because both have demonstrated that the kind and amount of behavioral training can have a profound and specific impact on the neuronal mechanisms involved in memory formation. In Drosophila, Tully and colleagues (1994) have shown that spaced and massed training sessions produce two forms of long-term memory that differ in their requirements of protein synthesis and sensitivity to genetic disruption. In addition, Mueller (1996) has shown in honeybees that $24 \mathrm{hr}$ retention after multiple conditioning trials requires the actions of nitric oxide synthase during training, whereas $24 \mathrm{hr}$ retention after a single conditioning trial does not. Thus, studies across different species provide converging evidence for a multiphasic view of long-term memory consolidation. These memory phases may be functionally independent and either separated or overlapping in time. Their expression depends on the duration, pattern, and intensity of training and thus may reflect an animal's ability to adapt optimally to the specific demands of unique environmental conditions.

\section{REFERENCES}

Abrams TW, Castellucci VF, Camardo JS, Kandel ER, Lloyd PE (1984) Two endogenous neuropeptides modulate the gill and siphon withdrawal reflex in Aplysia by means of presynaptic facilitation involving cyclic AMP-dependent closure of a serotonin-sensitive potassium channel. Proc Natl Acad Sci USA 81:7956-7960.

Ambron RT, Goldman JE, Schwartz JH (1974) Axonal transport of newly synthesized glycoproteins in a single identified neuron of Aplysia californica. J Cell Biol 61:665-675.

Ambron RT, Schmied R, Huang C, Smedman M (1992) A signal sequence mediates the retrograde transport of proteins from the axon periphery to the cell body and then into the nucleus. J Neurosci 12:2813-2818.
Atkinson RC, Shiffrin RM (1968) A proposed system and its control processes. In: The psychology of learning and motivation: advances in research and theory, Vol 2 (Spence KW, Spence JT, eds), pp 89-195. New York: Academic.

Bailey CH, Chen M (1988) Long-term sensitization in Aplysia increases the number of presynaptic contacts onto the identified gill motor neuron L7. Proc Natl Acad Sci USA 85:9356-9359.

Brunelli M, Castellucci V, Kandel ER (1976) Synaptic facilitation and behavioral sensitization in Aplysia. Possible role of serotonin and cyclic AMP. Science 194:1178-1180.

Byrne JH (1987) Cellular analysis of associative conditioning. Physiol Rev 67:329-439.

Byrne JH, Kandel ER (1996) Presynaptic facilitation revisited: state and time dependence. J Neurosci 16:425-435.

Carew TJ (1996) Molecular enhancement of memory formation. Neuron 16:5-8.

Carew TJ, Sahley CL (1986) Invertebrate learning and memory: from behavior to molecules. Annu Rev Neurosci 9:435-487.

Carew TJ, Castellucci VF, Kandel ER (1971) An analysis of dishabituation and sensitization of the gill-withdrawal reflex in Aplysia. Int J Neurosci 2:79-98.

Clark GA, Kandel ER (1993) Induction of long-term facilitation in Aplysia siphon sensory neurons by local application of serotonin to remote synapses. Proc Natl Acad Sci USA 90:11411-11415.

DeZazzo J, Tully T (1995) Dissection of memory formation: from behavioral pharmacology to molecular genetics. Trends Neurosci 18:212-218.

Dudai Y (1989) The neurobiology of memory: concept, findings, trends. Oxford: Oxford UP.

Emptage NJ, Carew TJ (1993) Long-term synaptic facilitation in the absence of short-term facilitation in Aplysia neurons. Science 262:253-256.

Frost WN, Castellucci VF, Hawkins RD, Kandel ER (1985) Monosynaptic connections from the sensory neurons of the gill- and siphon-withdrawal reflex in Aplysia participate in the storage of long-term memory for sensitization. Proc Natl Acad Sci USA 82:8266-8269.

Ghirardi M, Montarolo PG, Kandel ER (1995) A novel intermediate stage in the transition between short- and long-term facilitation induced by $5 \mathrm{HT}$ in the sensory-to-motor neuron synapse of Aplysia. Neuron 14:413-420.

Gibbs ME, Ng KT (1979) Behavioral stages in memory formation. Neurosci Lett 13:279-283.

Glanzman DL, Mackey SL, Hawkins RD, Dyke AM, Lloyd PE, Kandel ER (1989) Depletion of serotonin in the nervous system of Aplysia reduces the behavioral enhancement of gill withdrawal as well as the heterosynaptic facilitation produced by tail shock. J Neurosci 9:4200-4213.

Goldman-Rakic P (1992) Working memory and the mind. Sci Am 267:110-117.

Hammer M, Menzel R (1995) Learning and memory in the honeybee. J Neurosci 15:1617-1630.

Hawkins RD, Clark GA, Kandel ER (1987) Cell biological studies of learning in simple vertebrate and invertebrate systems. In: Handbook of physiology, Sec I, Higher functions of the nervous system (Plum F, ed), pp 25-83. Bethesda, MD: American Physiological Society.

Kamin LJ (1957) The retention of an independently learned avoidance response. J Comp Physiol Psychol 50:457-460.

Mauelshagen J, Carew TJ (1996) Temporal expression of 5HTinduced facilitation at Aplysia tail sensory-motor synapses. In: Brain and evolution. Proceedings of the 24th Goettingen neurobiology conference (Elsner N, Schnitzler HU, eds), p 557. Stuttgart: Thieme Verlag.

Mauelshagen J, Parker GR, Carew TJ (1995) Dynamics of induction and expression of long-term synaptic facilitation in Aplysia. Soc Neurosci Abstr 21:570.2.

Mauelshagen J, Sherff CM, Carew TJ (1996) Long-term facilitation in Aplysia sensory neurons: effects of massed vs spaced training and inhibition of synaptic transmission. Soc Neurosci Abstr 22:1405.

McGaugh JL (1966) Time-dependent processes in memory storage. Science 153:1351-1358.

Mercer AR, Emptage NJ, Carew TJ (1991) Pharmacological dissociation of modulatory effects of serotonin in Aplysia sensory neurons. Science 254:1811-1813. 
Montarolo PG, Goelet P, Castellucci VF, Morgan J, Kandel ER, Schacher S (1986) A critical period for macromolecular synthesis in long-term heterosynaptic facilitation in Aplysia. Science 234:1249-1254.

Mueller U (1996) Inhibition of nitric oxide synthase impairs a distinct form of long-term memory in the honeybee, Apis mellifera. Neuron 16:141-149.

Pinsker HM, Hening WA, Carew TJ, Kandel ER (1973) Long-term sensitization of a defensive withdrawal reflex in Aplysia. Science 184:1039-1042.

Rosenzweig MR, Bennett EL (1984) Direct processes and modulatory influences in the stages of memory formation. In: Neurobiology of learning and memory (Lynch G, McGaugh JL, Weinberger NM, eds), pp 263-288. New York: Guilford.
Squire L (1987) Memory and brain. New York: Oxford UP.

Stark LL, Mercer AR, Emptage NJ, Carew TJ (1996) Pharmacological and kinetic characterization of two functional classes of serotonergic modulation in Aplysia sensory neurons. J Neurophysiol 75:855-866.

Steward O, Banker GA (1992) Getting the message from the gene to the synapse: sorting and intracellular transport of RNA in neurons. Trends Neurosci 15:180-186.

Tully T, Preat T, Boynton SC, DelVecchio M (1994) Genetic dissection of consolidated memory in Drosophila. Cell 79:35-47.

Walters ET, Byrne JH, Carew TJ, Kandel ER (1983) Mechanoafferent neurons innervating the tail of Aplysia. II. Modulation by sensitizing stimulation. J Neurophysiol 50:1543-1559. 